In Crescendo. Institucional. 2015; 6(2): 153-168

Fecha de recepción: 25 de octubre de 2015

Fecha de aceptación: 10 de noviembre de 2015

\title{
SENTIDO Y ORIENTACIÓN DE LA POLÍTICA ACADÉMICA UNIVERSITARIA EN UN
}

CONTEXTO GLOBAL

\author{
SENSE AND GUIDANCE OF THE POLITIC ACADEMIC UNIVERSiTARY IN THE \\ GLOBAL CONTEXT
}

Áurea E. Rafael*

\section{RESUMEN}

T a decisión de analizar el sentido y perspectivas de las políticas académicas universiLtarias desde los ámbitos locales y globales, nos lleva a profundizar en el estudio de variables asociadas a la acción formadora en su interacción con la sociedad en cuanto a sus necesidades de crecimiento y desarrollo desde la educación superior. De esta forma se genera un diálogo entre el pensamiento y experiencia en gestión académica de Manel Viader, experto en temas de gestión universitaria de la Universidad de Barcelona, y las orientaciones de política en países de América Latina y el Caribe, en particular del Perú por contar con mayores precisiones conceptuales, regulaciones y modelos de gestión que ameritan ser revisados y mejorados de manera continua, a fin de sumar a sus impactos locales, nuevos logros regionales y globales. Se aborda una reflexión en torno a tensiones marcadas en el mundo universitario sobre la democratización-masificación, las estructuras organizativas y su relación con la función académica, la caracterizaciones de las titulaciones, organización y calidad docente, las metodologías de enseñanza aprendizaje, la evaluación en su nuevo paradigma de gestión, todo ello, en el marco de una cultura de cambio de las organizaciones universitarias, a modo de elementos clave en la política académica.

Palabras clave: política académica, cultura de cambio

\footnotetext{
* Docente principal de la Uladech Católica, Facultad de Educación.
} 


\begin{abstract}
The decision to analyse the sense and perspectives of the university academic politics from the local and global ambiences, leads us to studying in depth the study of variables associated with the forming action in its interaction with the society as for its needs for growth and development from the higher education.

Generating a dialogue between the thought and experience in academic management of Manel Viader, expert in topics of university management of the University of Barcelona and, the politics orientations in countries of Latin America and the Caribbean, in particular of Peru for being provided with major conceptual precisions, regulations and models of management who deserve to be checked and improved in a continuous way, in order to add up to its local impacts, new regional and global achievements.

A reflection is tackled concerning tensions marked in the university world on the democratization-overcrowding, the organizational structures and its relation with the academic function, the characterizations of the qualifications, organization and teaching quality, the methodologies of education learning, the evaluation in its new management paradigm, all this, in the frame of a culture of change of the university organizations.
\end{abstract}

Keywords: academic politics, culture of change

\title{
INTRODUCCIÓN
}

Los cambios profundos que vienen experimentando las universidades del mundo en cuanto a su concepción, organización, funcionamiento y rol social, nos invitan a reflexionar acerca de sus estilos e impactos de la formación de sus estudiantes en contexto. Pues el ámbito de intervención de los egresados universitarios, va dejando solo de ser local, para pasar a desempeños en ámbito socio cultural y geográfico más diversos; ello hace que la universidad desarrolle su visión glocal, espacio que combina lo global conjuntamente con lo local.

Siguiendo la línea de análisis de Viader en el contexto de la educación superior en España, se asume como variables la gestión académica, su dinámica e impactos en cuanto a calidad de sus procesos formativos considerando los fenómenos y cambios que imponen las tendencias globales en cuanto a calidad, innovación y producción de conocimiento, sin descuidar las necesidades de crecimiento y desarrollo de los contextos locales, a los cuales debe atender la universidad a través de sus egresados y los resultados de las investigaciones que proyecta.

En este sentido, la interacción entre universidad y sociedad cada vez es más dinámica y comprometida con las organizaciones que recepcionan a sus productos. Este hecho está ubicando a la universidad en un sitial de producción e innovación de conocimiento, a 
través de la acción curricular, el ejercicio docente y el rol estudiantil más comprometido con sus entornos sociales.

Por ello, el referente de las titulaciones y grados académicos pasan por mecanismos de garantía de la calidad a fin de lograr que los egresados manifiesten sus competencias profesionales en diversos entornos y circunstancias con plena satisfacción de sus demandantes laborales. Por tanto, la política académica debe ser la orientadora, la guía del desarrollo de los aprendizajes, pasando por la calidad mínima exigida al docente, quien se convierte en el gestor de los aprendizajes en diversos entornos, presencial, distancia o mixtos.

\section{DESARROLLO TEMÁTICO}

Vista la universidad como un elemento del sistema educativo nos aporta a una mejor comprensión de su presencia y rol fundamental en el desarrollo de la sociedad. En esta orientación, la universidad hace visible su capacidad para aportar a la transformación y mejora de la calidad de vida de los pueblos, a través de las personas que en ella se forman, como además de los resultados de sus investigaciones producto del accionar científico con el contexto global y/o local.

En este sentido, las interacciones entre la universidad y la sociedad se van fortaleciendo, a su vez que, la capacidad de respuesta de esta entidad ante las demandas y retos sociales se hace cada vez más latente. Mucho va a depender en esta relación del grado de implicación de la universidad con las necesidades de crecimiento social global; vale decir, en qué medida la política universitaria se orienta proactiva y responsablemente hacia la acción social aportando con conocimiento a los procesos de cambio ineludibles ante la búsqueda de crecimiento económico de los sectores empresariales lo cual trae consigo asumir, entre otros, nuevas formas de comunicación social, quiebres culturales y hasta en algunos casos, pérdida de identidad.

En este contexto, nos acercamos a través de estas reflexiones al análisis de situaciones de posicionamiento de la universidad a partir de sus políticas académicas, a fin de identificar y describir sus formas de organización, su capacidad de proyección en espacios no solo locales sino además internacionales, determinar el nivel de flexibilización de la oferta formativa y sus modalidades y, sobre todo, poner el énfasis en la gestión del aprendizaje sin desligar obviamente del ejercicio docente.

Tomando posición sobre lo dicho por Tedesco, con respecto al término "globalización": "Al estar basada fundamentalmente en la lógica económica y en la expansión del mercado, la globalización rompe los compromisos locales y las formas habituales de solidaridad y de cohesión con nuestros semejantes. Las élites que actúan a nivel global tienden a comportarse sin compromisos con los destinos de las personas afectadas por las consecuencias de la globalización. La respuesta a este comportamiento por parte de 
los que quedan excluidos de la globalización es el refugio en la identidad local donde la cohesión del grupo se apoya en el rechazo a los externos" (Tedesco, 2000). Queda claro que a la educación superior le corresponde participar en estos procesos de afrontamiento del fenómeno, mediante aporte de conocimiento que fortalezca la identidad y se pueda hacer frente a la expansión del mercado global y a su vez, poner en alerta el destino de las sociedades, básicamente de las emergentes, las cuales deben potencializarse en cuanto a su capital intelectual derivado de la puesta en marcha de procesos de gestión universitaria innovadoras centradas en el factor académico y en particular, en el estudiante y su contexto como escenario de su realización.

Las profundas transformaciones que se vienen dando en Latinoamérica como consecuencia de los procesos de globalización, propician una serie de cambios sociales, institucionales y económicas, tales como las reformas en los Estados, apertura a los mercados financieros, incremento de la tercerización productiva, entre otras, que tienen como consecuencia la segregación y marginalización social progresiva, que no permite a ciertos sectores sociales incorporarse a las dinámicas productivas y de desarrollo, no escapa de esta tendencia la universidad en su rol de productora de conocimiento y de espacio de debate ante las nuevas políticas educativas que se derivan de estos procesos globalizadores.

Esta situación impactante en el desarrollo social, hace que dirijamos una mirada más exhaustiva a los posibles efectos de la implantación de nuevas formas de organización de la universidad, analizar sobre la pertinencia de sus programas de formación, su nivel de participación y compromiso en el desarrollo de la ciencia y la tecnología. Asimismo, es necesario reparar en las implicancias y conveniencias de un sistema de formación por competencias, la naturaleza de los planes curriculares, el sistema de créditos, entre otros temas relacionados con estas políticas académicas que están sujetas a procesos de globalización.

Sobre estos temas se ha tenido la oportunidad de dialogar con el Manel Viader, experto en temas de políticas académicas en el contexto universitario de la comunidad europea; quien aporta al análisis y comprensión de variables asociadas al hecho académico en la Universidad de Barcelona UB, conocimiento base para desarrollar propuestas de integración a partir de un análisis de las políticas académicas en universidades latinoamericanas en el marco de la globalización, considerando nuevas tendencias y retos.

La universidad de hoy es una institución en cambio y Viader nos muestra a través de sus reflexiones de estos procesos y sus efectos; así como su aporte al relatar experiencias institucionales sobre afrontamiento al cambio y cómo las políticas académicas implementadas en universidades europeas vienen contribuyendo al desarrollo y gestión de conocimientos. Ubica en el centro de su reflexión a la UB, en razón de haber desempeñado en algún tiempo cargos de alta responsabilidad académica.

\section{Democratización-masificación}

Los actuales estatutos que rigen el funcionamiento y dinámica de las universidades vienen considerando la participación de la comunidad conformada por estamentos en los cuales el estudiante asume un rol de decisión en procesos electorales, en la dinámica 
académica al dar voz sobre sus satisfacciones frente a la calidad de formación recibida, así como en la representación externa ante otras comunidades educativas u organizaciones. Es decir, se viene fortaleciendo el espíritu democrático en las universidades. Tales es el caso, del sistema universitario peruano que por Ley Universitaria n. ${ }^{\circ} 30220$ del 2014, determinar el voto universal para la elección de las autoridades universitarias. Esta ley en la $1^{\circ}$ disposición transitoria dice "La elección se realiza mediante voto universal obligatorio y secreto de cada una de las categorías de los profesores indicados y por los estudiantes regulares $[\ldots] "$.

\section{Elementos clave en la política académica}

\section{Titulaciones}

Las titulaciones son reconocimientos oficiales de los títulos de grado que se emiten al finalizar una carrera universitaria, cuya duración en años la determinan los sistemas universitarios en cada país. Es preciso señalar que existe la posibilidad de establecer equivalencias de grados entre sistemas universitarios de países que establecen acuerdos o convenios.

Estas titulaciones se emiten de acuerdo a los niveles de estudios cursados, que pueden ser de grado (bachiller) y posgrado (maestría y doctorado). Estas titulaciones guardan correspondencia a las ofertas ocupacionales y salidas de profesionales, formando parte de catálogos oficiales de titulaciones de carreras que imparten las universidades; haciendo de manifiesto que el mercado de trabajo, un espacio en continua transformación, es el principal demandante de las carreras profesionales que se encargarán de formar al recurso humano especializado científica, técnica y/o humanísticamente.

Actualmente, el factor empleabilidad se asocia a la variable calidad de la formación profesional; por lo que las titulaciones y sus respectivos planes curriculares juegan un papel preponderante para el egresado, quien debe hacer evidente sus competencias alcanzadas en su etapa de formación académica. Por tanto, la titulación da cuenta de esas competencias logradas por sus egresados.

En el caso de la Universidad de Barcelona, las titulaciones universitarias de carácter oficial se estructuran en tres ciclos, cada uno de los cuales conduce a la obtención del título correspondiente. El primer ciclo conduce a un título de grado, y el segundo y tercer ciclo permiten obtener los títulos de máster y de doctorado, ambos comprendidos en lo que se conoce como programa de posgrado; el segundo ciclo conduce a la obtención del título de máster. A diferencia de los másteres ofertados hasta ahora, estos tienen carácter oficial, están reconocidos formalmente tanto en España como en el resto de países europeos, y tienen precios públicos, regulados por la Generalitat de Cataluña.

El máster universitario ofrece una formación de alto nivel, especializada tanto en un área específica del conocimiento como en áreas interdisciplinarias, con el objetivo de mejorar las competencias específicas en algún ámbito profesional o disciplinario. Entre los 
ámbitos profesionales a los que se dirigen, está la investigación. Por eso, el máster podrá integrarse como parte de la formación para la obtención del título de doctor.

Los másteres tienen una extensión de 60 a 120 créditos europeos y están dedicados a la formación avanzada, de carácter especializado o multidisciplinario, dirigida a una especialización académica o profesional, o bien a promover la iniciación en tareas de investigación. Muchos másteres son intercentros y algunos son interuniversitarios.

El tercer ciclo tiene como finalidad la formación avanzada del estudiante en las técnicas de investigación y conduce a la obtención del título de doctorado.

El doctorado es el estudio de posgrado al cual se puede acceder, como criterio general, cursando previamente estudios de máster universitario (periodo formativo del doctorado). Este máster tiene que permitir el acceso al programa de doctorado para poder entrar en el segundo periodo (de investigación), en el que se tiene que elaborar la tesis doctoral. La duración depende del tiempo necesario para hacer la tesis (Universidad de Barcelona, s. f.).

En América Latina y el Caribe (ALC) existe una gran diversidad de titulaciones. Cada país adopta su propio sistema de oferta de carreras profesionales, de tipos y niveles de estudios. Cuando el estudiante pretende hacer válido su título, ya sea para obtener un empleo o para continuar otros estudios profesionales en otro país de la región, surge el inconveniente de la falta de no convergencia entre las instituciones que lo expiden, en muchos casos este no es aceptado, lo que frena en primera instancia la movilidad del profesional, generando un obstáculo importante para establecer la cooperación con las universidades de la región y más con universidades de Europa.

A fin de que las titulaciones que brindan las universidades de ALC sean más acordes a la oferta de estudios y títulos de grado o posgrado europeos, debe lograrse una homologación de estudios y titulaciones, de tal manera que permita la movilidad entre los países de la región ALC y con la Unión Europea, iniciando de esta manera un proceso de cooperación educativa que en la actualidad es ineludible en atención a los mecanismos globalizantes y de integración cultural, cuyas implicaciones conducirían a un acercamiento entre sistemas educativos del nivel superior creando un espacio común intercontinental, emulando aspectos básicos del proceso de Bolonia, diríamos que se abrirían nuevas posibilidades para desarrollar programas de convergencia y acuerdos que favorezcan el desarrollo de la educación superior birregional.

Con referencia a la duración de las carreras en Europa se precisan de diferente manera los tres ciclos, por lo que implicaría que en América Latina las carreras se adecuen al tiempo de duración que se presenta en la estructura universitaria europea, aunado a la necesidad que existe de recortar el tiempo de estudio para que los estudiantes que culminen puedan acceder al mercado de trabajo con los conocimientos y competencias requeridas en ese momento y evitar que queden rezagados en virtud de la gran velocidad con que se genera actualmente el conocimiento. López Segrera (2007: 26) señala que para el año 2020 el conocimiento se duplicará cada 73 días, por lo que si no se realizan proyectos 
en tiempo y forma entre las naciones latinoamericanas para estar en sintonía con lo que acontece en Europa, estará muy lejana la concreción de lo establecido en el proyecto «orizonte 2015».

\section{Organización y procesos académicos}

La compleja estructura organizativa de la universidad (facultades, departamentos, escuelas, programas, etc.), así como el proceso de elaboración de los planes de estudio de carreras, en muchos casos con una diversidad de denominaciones de las experiencias curriculares para un mismo perfil profesional, trae dificultades a la concreción de programas de movilidad académica. Si esta situación se analiza desde la perspectiva de ganancia de conocimiento, se diría que es una barrera que impide el intercambio en una sociedad global que busca a todas luces generar capital intelectual que posibilite atender los requerimientos sociales y productivos. Todo ello analizado desde la cambiante perspectiva que aporta la sociedad del conocimiento.

De otro lado, las estructuras organizacionales rígidas y flexibles de las universidades, la oferta de modalidades de formación virtual, a distancia o semipresenciales, la conformación de coaliciones, consorcios, redes, conducen a la búsqueda de respuestas o alternativas a las demandas de formación de calidad, en donde los procesos académicos son fundamentales para alcanzar mejores respuestas. Por tanto, las unidades académicas deben ser gestionadas bajo un enfoque más dinámico y más acorde a los continuos cambios del entorno, debiendo modernizar, en muchos casos, sus currículos, el modelo de administración de sus recursos, así como sus procesos de control interno y externo.

\section{Organización docente}

El cuerpo docente de las universidades debe responder a las demandas de formación profesional a través de la conducción de procesos académicos orientados a la gestión de conocimientos, articulando la enseñanza-aprendizaje a la investigación y la proyección social. Ello exige, por tanto, que la docencia debe constituir redes de colaboración académica entre universidades o compartir proyectos de envergadura global.

Por otro lado, el reconocimiento a los méritos académicos docentes constituye un factor importante que aporta a la movilidad y a la conformación de comunidades científicas, que redundará en beneficio de la calidad formativa; es decir, la implementación de políticas universitarias orientadas a incentivar la producción de conocimiento bajo el liderazgo docente constituye un factor importante para elevar la calidad de los programas académicos.

En cuanto a las metodologías de enseñanza y aprendizaje que dinamizan el ejercicio docente a favor del estudiante, estas responden al logro de competencias profesionales mediante la interacción de estrategias movilizadoras de capacidades respetando los talentos, la diversidad de pensamiento y aspiraciones de los estudiantes, así como las características del contexto y tendencias de la profesión. Por tanto, la política debe pautar la ruta curricular en cuanto a las metodologías activas, participativas y conectadas al contexto, 
posibilitando al estudiante crecer en conocimiento y habilidades con pertinencia a las demandas de los sectores sociales y productivos de su región o de otras, donde el aprendizaje logrado facilitará la transferencia del conocimiento.

Los innovadores modelos de formación profesionales, requieren que los docentes asuman nuevos paradigmas de evaluación o de constatación de logros, partiendo por la autoevaluación como estrategia para validar sus propios esfuerzos para aprender (estudiante) y para enseñar (docente). En cuanto a la evaluación de los aprendizajes, la política debe orientar modelos centrados en las competencias, donde la evidencia o demostración del logro académico debe ser la pauta para la toma de decisiones de mejora académica.

Esto sugiere una transformación radical del sistema educativo que responde al cambio al que estamos asistiendo en las últimas décadas hacia una sociedad del conocimiento entendida también como una sociedad del aprendizaje. Se tendría que contar en cada nación de América Latina y el Caribe con un sistema de evaluación y, que dependan de una red latinoamericana para asegurar su coordinación, con el propósito de estar en sintonía con el sistema de evaluación europeo.

En cuanto a la inclusión progresiva de las TIC en la sociedad actual ha propiciado cambios en las actitudes y los comportamientos de las personas jóvenes y ha generado nuevas formas de comunicarse, de sentir y de vivir, que exigen replanteamientos a la pedagogía universitaria. En este sentido, se debe brindar atención al papel de las TICs en los procesos formativos, el docente debe poseer las habilidades para gestionar sus espacios pedagógicos utilizando soportes o recursos interactivos, atendiendo a la disponibilidad de medios tecnológicos que contribuyen al logro de aprendizajes en diferentes contextos, presenciales o no presenciales, reales o simulados.

En cuanto al desempeño de la docencia universitaria, este factor cobra especial atención dado que la sociedad en su conjunto exige calidad en el funcionamiento de las vinculándolas a criterios de eficacia, eficiencia y excelencia cada vez mayores (Arranz, 2007: 34), hecho que implica que la calidad ocupe un eje central en el diseño de la política universitaria. Es por ello que, la actuación del docente debe proporcionar satisfacción en sus estudiantes, al demostrar poseer las competencias y habilidades que favorezcan la mediación entre el estudiante y el nuevo saber, proceso en el cual las metodologías asociadas a la capacidad innovadora del docente son determinantes, muchas veces, en el logro de los perfiles académicos de los estudiantes.

De acuerdo con Marqués (2008), podemos afirmar que el EEES ha favorecido el interés hacia métodos pedagógicos innovadores. Por otro lado, Castilla (2011) refiere que "[...] La calidad ocupa un papel estratégico dentro de las diferentes normativas que regulan la actividad de las universidades españolas. La Ley Orgánica de Universidades (LOU) modificada en 2007, se refiere entre otras cuestiones en su artículo 31 a que "la promoción y la garantía de la calidad de las Universidades Españolas, en el ámbito nacional e internacional, es un fin esencial de la política universitaria", teniendo lo anterior objetivos como el mencionado en el apdo. c "la mejora de la actividad docente e investigadora y de la gestión de las Universidades"... De lo anterior se deduce la importancia de que las 
Universidades apuesten por la mejora de su calidad en términos generales y específicos para su labor docente [...]" (Castilla, 2011).

En cuanto a la calidad docente en ALC, el gran desafío en la región debe responder a ciertos condicionamientos que generan tensión, como son las de satisfacer las demandas sociales por una educación de calidad, de pasar de una concepción de educación tradicional centrada en el docente a un paradigma de formación centrado en el estudiante, de avanzar al ritmo de los cambios científico-tecnológicos, de implementar y ensayar creativamente nuevas formas de enseñar para aprender significativamente y de ajustarse a las restricciones en cuanto a recursos disponibles.

Sobre la docencia, Gonzalez (1990) nos dice que "Se entiende por docencia la actividad central, eje, en el proceso de formación, capacitación y actualización de los miembros de la comunidad académica, cuya médula es el proceso de enseñanza-aprendizaje. Percibida en forma específica, la docencia se comprende como un proceso organizado, intencionado y sistemático, a través del cual se promueven, se dirigen, se conducen o se facilitan aprendizajes significativos y acreditables". Dada la importancia del rol docente en las universidades y su gestión debe conducir al logro de los perfiles profesionales en sus estudiantes, es que en sociedades educativas tales como en Colombia, la calidad, la evaluación y el mejoramiento continuo constituyen la justificación del nuevo ordenamiento jurídico del sector. El primer principio sobre el que se fundamenta el Sistema de Educación Superior es "la excelencia académica como la búsqueda de altos niveles de calidad" (Ley Universitaria de Colombia, art. 6); este principio, obviamente, asocia calidad a excelencia académica y en este factor, el papel de la docencia se orienta a brindar calidad a los aprendizajes a través del uso de metodologías innovadoras que conjugan nuevos espacios instruccionales, entre ellas la educación a distancia y virtual.

En el Perú, el modelo de evaluación de la calidad para la acreditación de carreras universitarias propuesto por el Sistema Nacional de Evaluación y Acreditación de la Calidad Educativa Sineace (2008), considera en la dimensión de formación profesional, al factor docente el cual establece como criterios de evaluación: "Los docentes tienen la experiencia y capacidad requeridas para el desarrollo de las actividades de investigación en la carrera profesional. Tienen estudios de posgrado del más alto nivel, cuyos grados son validados y reconocidos por la autoridad peruana competente. Los docentes difunden su producción intelectual en revistas indizadas de su especialidad, a través de libros y como ponentes en congresos, seminarios y otros eventos nacionales e internacionales. La producción intelectual de los docentes (tesis, patentes, publicaciones en revistas o libros, etc.), está protegida mediante normas y procedimientos, para su reconocimiento dentro de la Universidad [...]". Estos criterios conducen a evaluar a través de un conjunto de estándares de calidad que buscan determinar el nivel de cumplimiento de las carreras en cuanto a la exigencia académica mínima de sus docentes, cuyos desempeños son sometidas a una valoración de sus estudiantes a través de encuestas de satisfacción, y de acuerdo con los resultados se adoptan las políticas de capacitación o actualización docente.

Con esta exigencia a la calidad de la docencia universitaria se busca contar con formadores no solo institucionales sino que compartan su ejercicio en nuevos espacios formati- 
vos en los ámbitos internacional y mundial; por ello, la política académica en cuanto a la docencia debe considerar los escenarios de internacionalización de la actividad formadora que propician la movilidad e intercambio docente. Pues es conveniente pasara de una cultura de desempeño docente local a una global.

\section{Docencia innovadora}

Las actuales reformas universitarias, consideran elementos tanto académicos como políticos; es decir sus planteamientos son integrales y contemplan medidas estratégicas así como operacionales. En lo que respecta al campo de la docencia, estas reformas inciden en el desarrollo de las capacidades innovadoras para diseñar y utilizar estrategias didácticas eficaces que promuevan en los estudiantes las competencias previstas en los planes curriculares, asociadas a la aplicación y renovación del conocimiento, a partir de la investigación que realiza el docente en interacción con sus pares, quienes pueden estar ubicados en espacios geográficos diferentes; pero que con ayuda de las tecnologías de la información y comunicación, hacen posible esta interactividad educativa; es decir, estamos frente a escenarios mediados por la innovación metodológica ante la necesidad de las universidades de adaptarse a un mundo global y a la sociedad del conocimiento a partir del cambio del modelo docente.

Fernández (2006) refiere que "[...] el saber es cada vez más extenso [...], el conocimiento presenta una tendencia a la fragmentación y especialización y, [...] el ritmo de producción de ese conocimiento es cada vez más acelerado y, por tanto, su obsolescencia también crece". Ante estas circunstancias es importante desarrollar en la docencia universitaria un pensamiento metodológico innovador que impacte en el ánimo e interés de sus estudiantes por aprender de manera duradera y selectiva, consciente de la utilidad de aprender, enfatizando más en capacidades y habilidades, que en contenidos disociados de su campo de aplicación.

\section{Docencia y aprendizaje}

En la Conferencia Mundial sobre la Educación Superior, la Unesco (2009) expresó la necesidad de nuevos enfoques que permitan a "la Educación Superior ampliar la formación de los profesores con planes y programas de estudios que den a los docentes la capacidad de dotar a sus alumnos de los conocimientos y las competencias que necesitan en el siglo XXI" (Unesco, 2009, p. 3). Sobre lo cual, Viader nos lleva a una reflexión sobre la acción mediadora del docente. El modelo tradicional de docencia universitaria basado en la transmisión del conocimiento frente a los cambios que introduce la sociedad del conocimiento y su impacto en nuevas dinámicas educativas en educación superior condiciones de enseñanza y aprendizaje en el acceso a la universidad. Se aborda la práctica docente universitaria desde su triple perspectiva: los materiales para el proceso educativo y sus condiciones, las estrategias pedagógicas y finalmente el papel de la organización institucional.

En este sentido, las políticas académicas conducen hacia propuestas educativas transformadoras y no reproductoras, con cambios sustanciales a nivel micro educativo que 
conllevan al estudiante a nuevos comportamientos sociales y laborales. Es decir, " ... la formación universitaria ha de favorecer un aprendizaje flexible, si bien regido por criterios fiables y justificados, planteando una visión del conocimiento como proceso constructivo, hecho este que permite vincular el aprendizaje con la propia investigación científica.." Fernández (2006).

\section{Modelos de formación}

Dar cuenta de la variedad de modelos educativos hasta hoy, no es una tarea fácil, en el sentido que se tienen experiencias formativas locales (muy particulares) y experiencias formativas internacionales (de convergencia). En una primera aproximación, entendemos que puede ser útil clasificarlas de acuerdo con tres dimensiones generales, a saber: La perspectiva o nivel en que se sitúan las acciones formativas, la organización y estructura de las mismas, y las modalidades de formación ofertadas o disponibles.

La diversidad de modelos de formación aportar mayor facilidad para cambiar de estudios dentro de tu universidad o en cualquier universidad. Si una vez comenzada una carrera y si se quiere cambiar a otra, se reconocerá más fácilmente lo aprobado en la primera- Posibilita además, una mayor facilidad para estudiar en el extranjero.

Ante las diferentes modalidades de formación innovadora, las políticas académicas universitarias deben considerarla dentro de las posibilidades de metodológicas para la gestión curricular, tal como lo determina la regulación universitaria peruana a través de la Ley Universitaria 30220 en su artículo 39: "El régimen de estudios se establece en el Estatuto de cada universidad, preferentemente bajo el sistema semestral, por créditos y con currículo flexible. Puede ser en la modalidad presencial, semipresencial o a distancia".

\section{Formación presencial}

Es el espacio tradicional donde interactúa cara a cara los estudiantes con el docente, comparten los propósitos de la formación, así como el espacio físico generando su propio contexto del aprendizaje. Por lo general, responde a una planificación de clases o sesiones de tiempo rígido, donde prima un único ritmo de aprendizaje. Favorece la aplicación de técnicas donde interviene la representación y la espontaneidad de los participantes, generando una incidencia importante del proceso motivacional.

Desde la perspectiva de Barberá (2014) "Un tutor muy experto quizá podría entrar en una clase presencial e impartir una excelente lección sin una mayor preparación anterior, incluso repetir el hecho indicado clase tras clase y entusiasmar a sus alumnos creando un buen clima de diálogo y discusión", lo cual constituiría en una de las bondades de este tipo de formación. En este sentido, la política académica debe considerar estas ventajas que aporta la formación presencial.

\section{Formación tutelada (planificada)}

Consiste en la conducción del aprendizaje de manera diferenciada e individualizada según las características, intereses y necesidades de cada participante; pero que responde 
a un programa formativo previsto. En esta modalidad, los docentes tutelan a los participantes, conduciéndoles progresivamente a lograr sus propósitos. Tal conducción (coaching) es efectuada por instructores provistos de amplio margen de flexibilidad y autonomía para el desempeño de sus actividades docentes, y con altísima experiencia en cuanto a los temas que se abordan (contenido), así como en la personalización del aprendizaje, sabiendo fomentar un clima de confianza y manteniendo un contacto directo, franco y amigable con cada participante (CIEG, s. f.).

La vinculación entre el instructor y el participante se hace totalmente a distancia, utilizando materiales de consulta y referencia sugeridos por el tutor, correo electrónico y medios de intercambio de archivos, voz y video, como Skype u otras alternativas disponibles.

\section{Formación e-learning}

Formación combinada, del inglés blended learning consiste en un proceso docente semipresencial; esto significa que un curso dictado en este formato incluirá tanto clases presenciales como actividades de e-learning.

Este modelo de formación hace uso de las ventajas de la formación 100\% on-line y la formación presencial, combinándolas en un solo tipo de formación que agiliza la labor tanto del formador como del alumno. El diseño instruccional del programa académico para el que se ha decidido adoptar una modalidad b-learning deberá incluir tanto actividades on-line como presenciales, pedagógicamente estructuradas, de modo que se facilite lograr el aprendizaje buscado.

Asumiendo la fundamentación de aprendizaje que aporta esta modalidad b-learning, "El aprendizaje puede ser visto como un proceso activo y constructivo a través del cual la persona que aprende manipula estratégicamente los recursos cognitivos disponibles de manera de crear nuevos conocimientos extrayendo la información del entorno e integrándola a la estructura de información ya presente en su memoria" (Lebrun, 2005), sentamos posición de que es una nueva forma de lograr aprendizajes, más allá de las ventajas que aporta la formación exclusivamente presencial.

En el caso del Cap. V "Organización universitaria" de la Ley Universitaria del Perú n. ${ }^{\circ}$ 30220, en su artículo 47: "Las universidades pueden desarrollar programas de educación a distancia, basados en entornos virtuales de aprendizaje. Los programas de educación a distancia deben tener los mismos estándares de calidad que las modalidades presenciales de formación. Para fines de homologación o revalidación en la modalidad de educación a distancia, los títulos o grados académicos otorgados por universidades o escuelas de educación superior extranjeras se rigen por lo dispuesto en la presente Ley", otorga posibilidades para potencializar la modalidad a distancia y la movilidad académica con universidades extranjeras.

El impulso del uso innovador de las tecnologías de la información y comunicación para definir nuevas posibilidades de aprendizaje en el marco de las titulaciones actuales y nuevos programas formativos para acceder a nuevos destinatarios, deben ser bien 
aprovechadas por esta modalidad muy flexible a los contextos de los estudiantes que no disponen de tiempos suficiente para recibir una formación exclusivamente presencial.

\section{Formación autónoma}

La formación autónoma se asocia al término autoaprendizaje, el cual hace referencia a aprender uno mismo en un acto auto reflexivo, de allí que para referirse al aprendizaje llevado a cabo por uno mismo, sea más adecuado utilizar el término aprendizaje autónomo.

"El ejercicio autónomo posibilita y estimula la creatividad, la necesidad de la observación, sin embargo su trabajo debe ser confrontado por todos los actores de la comunidad educativa e incluso por la sociedad en la cual interactúa. En este tipo de formación el individuo debe estar preparado y sobre todo, abierto a la dinámica de la evolución de los saberes y al avance de las investigaciones en todas las áreas, es por ello, que el aprendizaje autónomo se convierte en una de las mejores herramientas del aprendizaje permanente para estar al día en el devenir progresivo de la vida misma" (Universidad Nacional Abierta y a Distancia) (González Sepúlveda, 2011).

Esta formación posibilita no solo aprender de su contexto inmediato, sino de otros contextos a decisión del programa o del propio aprendiz.

\section{Organización docente}

El equipo docente (definido, identificado, reconocido e incentivado) como base de la organización de la actividad formativa, generador de un entorno adecuado para la mejora de la calidad del aprendizaje, despliega un rol importante en la política académica. Por ello, debe aprovecharse la competencias y potencialidades de este recurso humano, tanto cualitativa como cuantitativamente; es decir contar con los más calificados profesionalmente y con la disponibilidad para tender a un número adecuado de estudiantes.

Los docentes universitarios se organizan por categorías y/o modalidades, por áreas de especialización o cuerpo de asignaturas, de acuerdo a las características de los planes de formación donde se desempeñan; por lo que la universidad debe crear el marco idóneo para impulsar sus iniciativas de innovación y perfeccionamiento académico, más allá de la asignatura concreta.

\section{Mejora, innovación y calidad}

Incrementar el impacto de la amplia actuación de mejora e innovación docente presente en la Universidad: Comunicación, institucionalización de buenas prácticas, creación de entornos innovadores.

Desarrollo profesional del profesorado universitario en el ámbito docente a través de un gabinete pedagógico, una escuela de docente, plan institucional de formación, indicadores de calidad docente, seguimiento sistemático; contrato de programa, planes de mejora docente; revisión profunda del sistema de evaluación de la actividad docente; reconocimiento y proyección de la docencia; reconocimiento de la excelencia. 
E-learnig aprendizaje on line es definida por la Fundación para el Desarrollo de la Función Social de las Comunicaciones (Fundesco) como: "Un sistema de impartición de formación a distancia, apoyado en las TIC que combina distintos elementos pedagógicos: Instrucción clásica (presencial o autoestudio), las prácticas, los contactos en tiempo real (presenciales, videoconferencias o chats) y los contactos diferidos (tutores, foros de debate, correo electrónico)" (Marcelo, 2002), generando un mundo de interacciones. Razón suficiente para pensar en la política académica debe asumir estas nuevas tendencias formativas, en las cuales las TIC serán de mediación obligatoria.

Sobre el tema Gewerc (2010) nos dice que "[...] podemos distinguir dos grandes tipos de estudio de interacciones. Por un lado, estudios experimentales en los que se llevan a cabo tareas específicamente y se analizan las interacciones usando algún esquema de codificación (datos cuantitativos) [...] Por otro, estudios observacionales del habla y de las interacciones de alumnos trabajando juntos en sus actividades usuales en clase, en las que se utilizan métodos cualitativos e interpretativos".

\section{CONCLUSIONES}

1. Debe perfeccionarse la capacidad de gestión universitaria en tópicos específicos y promover el uso de nuevas técnicas y elementos pedagógicos en la función docente de las instituciones de educación superior.

2. Además de la evaluación, la investigación es un mecanismo que puede facilitar el análisis de la situación actual y de las líneas futuras de la Formación profesional Universitaria FPU (Zabalza, 2009).

3. Es necesario promover la investigación en el campo de la gestión académica, para identificar modelos de formación innovadores, valorar su impacto, reconocer necesidades y definir posibles escenarios donde se moverá a futuro el sistema universitario.

4. La tendencia hacia una cierta reformulación del concepto global de formación universitaria desde la perspectiva de la flexibilidad y la personalización de los currículos, conlleva a repensar y revisar continuamente los impactos de la política académica.

5. Debe destacarse el valor muy importante de las fórmulas mixtas (blended learning) para incluir en los programas de formación académica, optimizando los espacios de presencialidad acorde a las tendencias pedagógicas contemporáneas y al perfil de competencias que hoy demanda desarrollar el entorno social. En este sentido, debe profundizarse la indagación sobre la interacción como medio de aprendizaje y sus impactos de los cambios tecnológicos, traídos desde la globalización y la inclusión de las TIC. 


\section{REFERENCIAS BIBLIOGRÁFICAS}

Aramburuzabala, P., Reyes H., Ángel-Uribe, I. (2013) Modelos y tendencias de la formación universitaria. Profesorado, 17(3).

Barberá, E. (s/f). Los fundamentos teóricos de la tutoría presencial y en línea: una perspectiva socioconstructivista. Educación en Red y Tutoría en Línea. Estudios de Psicología y Ciencias de la Educación. Universitat Oberta de Catalunya .

Castilla, Francisca. (2011). En Educade 2, 2011, p. 170

CIEG (S. f.) http://www.grupocieg.org/archivos/Tr\%C3\%ADptico\%20Liderazgo\%20Femenino\%20\%28tutelar\%29.pdf Recuperado el 6 de mayo 2015.

Cinda. (1990). Calidad de la docencia universitaria en América Latina y el Caribe. Alfabeta Impresores, Chile.

Fernández, A. (2006). Educatio siglo XXI, 24· pp. 35-56.

Gewerc, A. (2010). El lugar de las TIC en la enseñanza universitaria. Estudio de caos en Iberoamérica. Málaga: Ediciones Aljibe.

Gewerc, Adriana. (Coordinadora). El lugar de las TIC en la enseñanza universitaria: estudio de casos en Iberoamérica, p. 42

Gómez, I. y García, F. (2014). Manual de didáctica. Aprender a enseñar. Madrid: Pirámide.

Gonzales Sepúlveda En http://datateca.unad.edu.co/contenidos/434206/434206/captulo 4 aprendizaje autnomo.html Recuperado el 4 de mayo del 2015.

Gonzalez, Eduardo. (1999). CINDA.

Lebrun, M. (2005). E- learning para enseñar y aprender. Vincular pedagogía y tecnología, Universidad Católica del Norte, Chile.

Artículo 3 de la Ley 30 de 1992, Colombia.

Miñano, B. y Rodríguez, J. (2011) Calidad, evaluación y acreditación en la educación superior: a propósito de un proyecto de reforma en Colombia. Revista Pensamiento Jurídico.

Unesco. (2003). Ministerial Round Table Meeting on Quality Education. Disponible: http://portal.unesco.org/en/ev.php-URL ID=15505\&URL DO=DO TOPIC\&URL $\underline{\text { SECTION=201.html }}$

Universidad de Barcelona (S. f.). En : http://www.ub.edu/web/ub/es/estudis/oferta formativa/graus/objectius estructura/grau noves titulacions/grau noves titulacions.html Recuperado el 6 de mayo 2015. 
Tedesco, Juan Carlos. (2000). Educación y sociedad del conocimiento y de la información. Revista Colombiana de la Educación 6.

Ley 30220. Ley Universitaria del Perú, capítulo V de la Organización Académica, artículo $39^{\circ}$.

Ley Universitaria 30220 de la República del Perú. En http://www.unmsm.edu.pel transparencia/archivos/NL20140709.PDF Recuperado 6/05/15.

Aprendizaje autónomo. En: http://datateca.unad.edu.co/contenidos/434206/434206/ captulo 4 aprendizaje autnomo.html. Recuperado 11/05/15.

SINEACE, CONEAU (2008). Modelo de calidad para la acreditación de carreras profesionales universitarias. 